\title{
Prevalence of gastrointestinal disorders recorded at postmortem inspection in white veal calves and associated risk factors
}

\author{
M. Brscic, ${ }^{* 1}$ L. F. M. Heutinck, $†$ M. Wolthuis-Fillerup,† N. Stockhofe,‡ B. Engel,§ E. K. Visser,† F. Gottardo, ${ }^{\star}$ \\ E. A. M. Bokkers,\# B. J. Lensink, || G. Cozzi, ${ }^{*}$ and C. G. Van Reenen† \\ *Department of Animal Science, University of Padova, Viale dell'Università 16, 35020 Legnaro (PD), Italy \\ †Livestock Research, Wageningen UR, PO Box 65, 8200 AB Lelystad, the Netherlands \\ $\neq$ Central Veterinary Institute, Wageningen UR, PO Box 65, 8200 AB Lelystad, the Netherlands \\ §Biometris, Wageningen UR, PO Box 16, $6700 \mathrm{AH}$ Wageningen, the Netherlands \\ \#Animal Production Systems, Wageningen UR, PO Box 338, $6700 \mathrm{AH}$ Wageningen, the Netherlands \\ ||Groupe ISA, 48 Boulevard Vauban, 59046 Lille Cedex, France
}

\section{ABSTRACT}

The study aimed at assessing the prevalence of poor rumen development, presence of rumen plaques, rumen papillae hyperkeratinization, and abomasal lesions in veal calves and to investigate risk factors for their occurrence at the farm level. Within a wide cross-sectional study, a sample of 170 veal farms representative of the European veal meat production systems was considered in the 3 major producing countries (99 in the Netherlands, 47 in France, and 24 in Italy). An average of $59 \pm$ 10 (SD) rumens and abomasa belonging to calves from a single batch per farm were inspected at the abattoir by trained observers to assess the incidence of these gastrointestinal disorders. Potential risk factors for their occurrence related to farm management, housing, and to the feeding plan were obtained by a questionnaire submitted to the stockperson. Prevalence of poor rumen development (almost no papillae present), rumen plaques, and hyperkeratinization were $60.4,31.4$, and $6.1 \%$ of rumens, respectively, whereas abomasal lesions in the pyloric area were recorded in $74.1 \%$ of abomasa. Independent variables related to the feeding system confirmed to be the main risk factors for the occurrence of gastrointestinal disorders in veal calves. However, additional risk sources for each given problem were identified among housing and management variables. The provision of a low amount of solid feed ( $\leq 50 \mathrm{~kg}$ of dry matter/head per cycle) was a relevant risk for rumen underdevelopment. Rumen wall alterations (plaques and hyperkeratinization) and abomasal lesions were instead associated with the administration of large quantities of solids (151-300 kg of dry matter/ head per cycle) in calves receiving milk replacer during the entire fattening cycle. Among the types of solid

Received May 27, 2010.

Accepted October 30, 2010.

${ }^{1}$ Corresponding author: marta.brscic@unipd.it feed, cereal grain acted as a preventive measure for low rumen development, whereas it was a risk factor for the occurrence of rumen plaques, papillae hyperkeratinization, and abomasal lesions. Some housing and management options adopted to improve veal calf welfare (i.e., higher space allowance and use of heating) were associated with lower risk for gastrointestinal disorders.

Key words: veal calf, gastrointestinal disorder, risk factor, welfare

\section{INTRODUCTION}

Worldwide, surplus male dairy calves are mainly used for veal production in specialized fattening units under intensive rearing conditions. Europe raises about 6 million calves per year for veal meat production and France, the Netherlands, and Italy are the main producing countries with a yearly production of $1.6,1.2$, and 0.9 million calves, respectively (EUROSTAT, 2007). Veal calf rearing systems are similar and very standardized across European Union countries. Nowadays, about $90 \%$ of the veal calves are housed in small pens and this housing system is mainly adopted in the Netherlands and Italy. The remaining farms house calves in larger groups of 30 to 60 animals and this housing solution is more frequent in France. Calves in small groups receive the milk replacer in individual buckets or in a common trough, whereas milk delivery in large groups is carried out through computer-controlled automatic milk delivery devices (AMD).

White veal calf production has been strongly criticized for poor animal welfare (Broom, 1991). To fulfill the physiological and behavioral demands of the calf, a specific European legislation for their protection set a ban on the individual cage and added the provision of solid feed in addition to the all-liquid diet (European Council, 1991, 1997; directives 91/629/EC and $97 / 2 / \mathrm{EC}$ ). Relevant improvements of calf behavior and productive performance were achieved by the veal 
producers after the introduction of the group housing (Andrighetto et al., 1999; Babu et al., 2004). Moreover, the compulsory provision of increasing amounts of solid feed in addition to the all-liquid diet reduced abnormal oral behaviors (Mattiello et al., 2002; Di Giancamillo et al., 2003) and promoted rumen development (Morisse et al., 2000; Suárez et al., 2006a).

Despite these recent advances in veal production, calf welfare is still compromised by gastrointestinal disorders such as rumen mucosa alterations and abomasal lesions (Cozzi et al., 2002a; Bähler et al., 2010). Hyperkeratinization, also called rumen parakeratosis, is one of these mucosa alterations in calves. It occurs when epithelial squamous cells develop a hardened keratin layer due to the diet's inability to continuously remove degenerating epithelial cells (Hinders and Owen, 1965). Parakeratosis creates a physical barrier, restricting absorptive surface area and VFA absorption, reducing epithelial blood flow and rumen motility, and causing papillae degeneration and sloughing (Beharka et al., 1998). Parakeratosis is often associated with concentrate diets with small particle size and low abrasive value (Greenwood et al., 1997), increased VFA production, decreased rumen buffering capacity, and subsequently decreased rumen pH (Anderson et al., 1982). A calf with parakeratosis might, therefore, be more easily subject to potential (sub)acidosis, which is detrimental to its welfare. Next to rumen parakeratosis, rumen plaques can be found in a certain number of cases, which means that feed particles become impacted in between the ruminal papillae (Suárez et al., 2006b). Although the exact consequences on the calf's health are still unknown, it can be imagined that the absorptive capacity of the rumen papillae is limited in the case of rumen plaques, with a possible accumulation of VFA in the rumen. Poor rumen development and the impaired integrity of rumen and abomasal mucosa are supposed to cause economic losses by decreasing VFA absorption and feed conversion efficiency. Abomasal lesions may also develop into perforating ulcers, which are one of the main causes of death in veal calves (Bähler et al., 2010).

The etiology of most of these disorders has been mainly related to the calf feeding plan and feed characteristics, as summarized in Figure 1. According to Suárez et al. (2006b) the incidence of rumen plaques was increased by the provision of concentrate diets in addition to the milk replacer. Abomasal lesions were suggested to be associated with the overloading of the stomach by the high volumes of milk replacer delivered in a small number of meals per day (Bokkers and Koene, 2001; Veissier et al., 2001), as well as to the mechanical damage caused by roughage with high fiber content (Wiepkema et al., 1987; Morisse et al., 2000).
However, other predisposing factors or causative stressors have also been reported as potential risk factors for the occurrence of this problem. Recent findings from Bähler et al. (2010) suggested that the high prevalence of abomasal lesions may be associated with risk factors such as the frequency of cleaning or the type of ventilation (manual versus mechanical) of the housing facility.

No on-field investigations have assessed how the prevalence of gastrointestinal disorders, such as low rumen development, rumen mucosa alterations, and abomasal lesions are affected by the different management factors of modern veal calf husbandry. Therefore, a large-scale risk factor analysis is needed to quantify the prevalence of these disorders as well as to identify their predisposing causes. These 2 goals were targeted in this research through a cross-sectional study on a large sample of veal calf farms in Europe.

\section{MATERIALS AND METHODS}

\section{Farm Sample}

A comprehensive cross-sectional study was carried out, in part, within the Welfare Quality project, with the joint involvement of research groups from the 3 main white veal meat-producing countries in Europe (the Netherlands, France, and Italy). Across the 3 countries, a sample of 170 veal calf farms was chosen to reflect the proportions of the prevailing rearing systems operating in Europe in terms of type of housing (small or large groups) and milk delivery strategies (bucket, trough, automatic feeding device). The sample consisted of 148 farms housing calves in small groups (92 farms in the Netherlands, 35 farms in France, and 21 farms in Italy), equipped either with individual buckets or a common trough for milk delivery. The remaining 22 farms (7 farms in the Netherlands, 12 farms in France, and 3 farms in Italy) housed animals in large groups adopting AMD. The sample within each country considered farms located in the main regions where veal calves are reared and it was selected among farms belonging to integrators/owners willing to participate in the study. A single batch of calves was considered for each farm, and the tested batches were evenly distributed across all 4 seasons.

A randomized group of calves belonging to the same batch per farm was followed at the slaughterhouse at the end of the fattening cycle. Inspected calves were a random subsample of the batch slaughtered on $1 \mathrm{~d}$, considering that 1 entire batch of veal calves could be slaughtered on several days and in different slaughterhouses. 


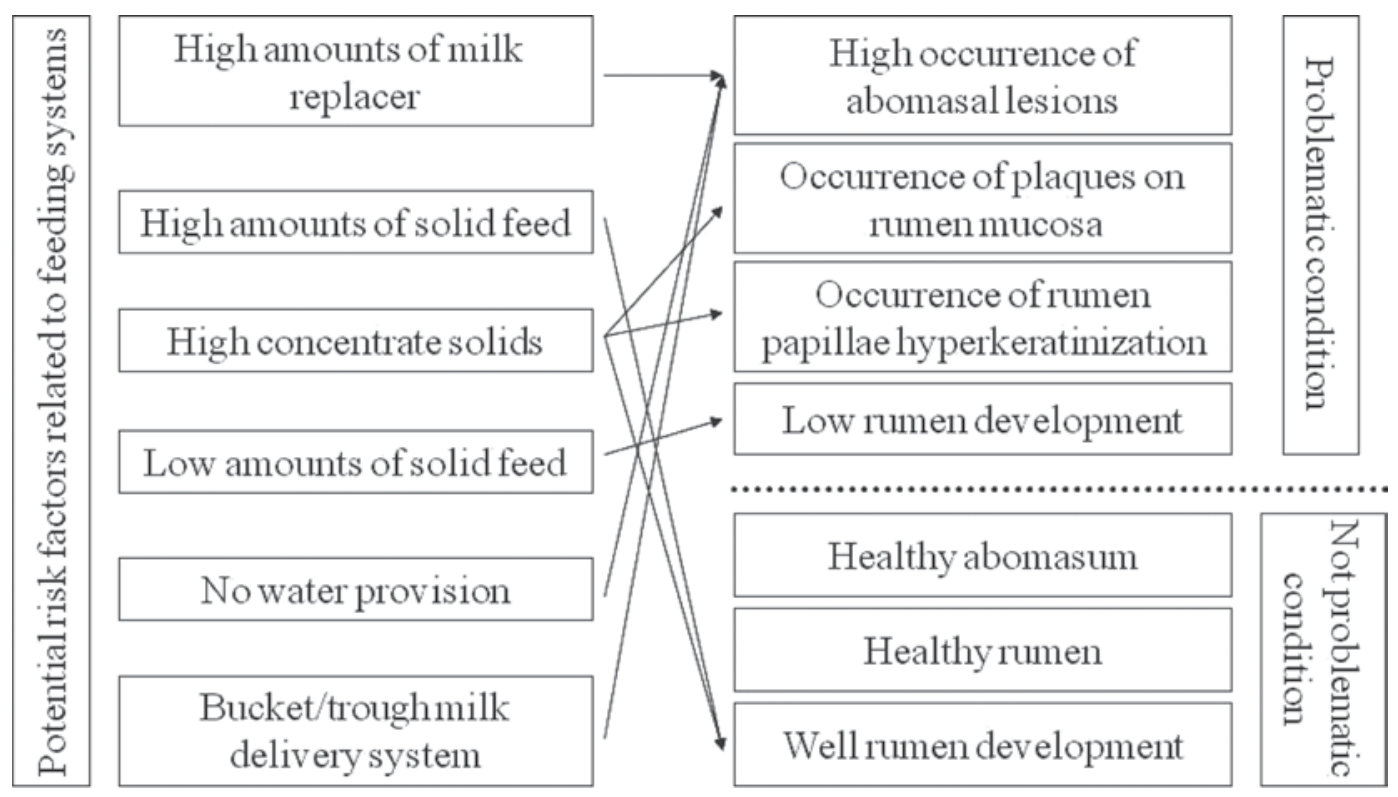

Figure 1. Schematic association between problematic or nonproblematic conditions of the digestive system of preweaned calves and effects related to the feeding strategies described in the literature (Wiepkema et al., 1987; Suárez et al., 2006b; Bähler et al., 2010) that were considered as the starting point for the hypothesis formulation and risk factors investigation in the present study.

\section{Farm Environment and Management Data}

Specific data regarding veal calves management, housing, and resources were gathered from an interview with the stockperson carried out on each farm. Data concerning the amounts of milk powder and prevalent type and quantity of solid feed delivered to calves throughout the fattening cycle were also provided by the farm manager. Duration of the rearing cycle was calculated as the difference between the week of slaughter and the week of arrival of the considered batch at the fattening unit.

\section{Postmortem Inspection}

At the abattoir, rumens and abomasa were examined by trained observers. Rumens were evaluated after opening and rinsing the organs in water. Rumen development was graded according to the following scale: score $1=$ low-developed rumen having almost no papillae in the atrium and in the ventral and dorsal rumen; score 2 = rumen with moderate development having few papillae in the ventral and dorsal rumen and the rumen wall still visible; score $3=$ well-developed rumen having a moderate number of papillae in the ventral and dorsal rumen, and rumen wall still visible but with numerous papillae in the atrium; and score 4 = fully developed rumen having numerous papillae in the ventral and dorsal rumen and with leaflet shaped papillae in the atrium.
The presence of plaques on the rumen wall or papillary epithelial hyperkeratinization were also recorded as a binary measure (yes/no). Rumen plaques consist of multiple patches on the mucosa with coalescing and adhering papillae covered by a sticky mass of feed, hair, and cell debris (Suárez et al., 2006b). Hyperkeratinization is characterized by papillae of thicker and harder texture. Visually, a hyperkeratinized epithelium looks like thickened and clustered papillae of a more rounded shape and its tissue is harder at physical contact.

Abomasa were evaluated in the last $15 \mathrm{~cm}$ of the pyloric area after opening it by a longitudinal cut. Presence of any kind of lesion at the mucosa (from erosion to open ulcer) was recorded as a binary measure at the pyloric area level and at the torus pylorus level. In accordance with the Welfare Quality Consortium protocol for veal calves (Welfare Quality Consortium, 2009 ), lesions in the pyloric area were counted from $0=$ absence of lesions to a censored maximum of $4=$ presence of 4 or more lesions within each of the following 3 size classes: $1=$ lesions with a diameter $<0.5 \mathrm{~cm}^{2}, 2$ $=$ lesions with a size between 0.5 and $1 \mathrm{~cm}^{2}$, and $3=$ lesions $>1 \mathrm{~cm}^{2}$.

\section{Data Processing and Statistical Analysis}

Raw data gathered during postmortem inspection were expressed as percentages of organs with a given score (e.g., \% rumens with score 1) or with a given problem (e.g., \% abomasa with at least one lesion on 
torus pylorus) over the total number of examined organs per farm. Moreover, a mean score was calculated per farm for both rumen development (mean of the scores attributed to all of the observed rumens) and abomasal lesions. To estimate the overall damage at the abomasums, a weighted lesions mean score was calculated as follows: [(number of lesions of size $1 \times 1)+$ (number of lesions size $2 \times 2$ ) + (number of lesions size $3 \times 3)$ ] and it ranged from 0 for no lesions to a censored maximum of 24 .

Data were statistically analyzed using GenStat (GenStat Committee, 2000), with farm as the experimental unit. Descriptive analyses were carried out on both response and explanatory variables. The response variable was one of the gastrointestinal disorders, whereas the explanatory variables were the potential risk factors obtained from the farmer interviews. The distribution of farms for each continuous risk factor (e.g., covariable, such as farm size or total amount of solid feed) included in the data set was displayed with the use of histograms. Subsequently, all covariables were transformed into class variables with a maximum of 4 classes. In the case of normally distributed covariables, thresholds for classes were chosen mainly according to location parameters such as lower quartile, median, and upper quartile. For nonnormally distributed covariables, thresholds were arbitrarily chosen in such a way that sufficient numbers of farms were present in each class. In the case of the covariable space allowance, a cut-off point equal to the minimum legal requirement of $1.8 \mathrm{~m}^{2}$ (for calves with a body weight over $220 \mathrm{~kg}$ ) could be conveniently applied. Thus, all potential risk factors were defined as discrete (class) variables. An overview of potential risk factors and corresponding levels is given in Table 1.

Risk factor analysis was carried out in a stepwise way. Each gastrointestinal disorder was analyzed separately and, consistent with a threshold suggested by Gillman et al. (2009), risk factor analysis was carried out only for problems that showed an average prevalence $>5 \%$. When the inspection of the same organ (rumen and abomasum) considered different classes of evaluation (e.g., rumen development) and the calculation of a mean score (e.g., rumen development and abomasal lesions), risk analysis was performed on the response variable most correlated with the others for the same organ (Spearman rank correlations) or to the most relevant according to the literature. Poor rumen development (score 1) was included in the risk analysis because it is reported in the literature as associated with poor calf welfare and it was highly negatively correlated with well-developed rumen $(\mathrm{r}=-0.80, P<$ $0.001)$ and full rumen development $(\mathrm{r}=-0.75, P<$
$0.001)$, as well as with the mean score $(\mathrm{r}=-0.99, P$ $<0.001$ ). Similarly, the percentage of abomasa with lesions in the pyloric area was highly positively correlated with the occurrence of lesions on the torus pylorus $(\mathrm{r}=0.62, P<0.001)$ and with the mean score $(\mathrm{r}=0.86, P<0.001)$. First, a univariate analysis was performed to study the effect on the dependent variable (Y) of each single factor separately using a generalized linear model. A 2-way ANOVA was then carried out to test for each pair of risk factors the significance of main effects and their interaction as well as to evaluate potential multicollinearity between risk factors through the inspection of the distribution of farms across combinations of risk factors, and the calculation of the variance inflation factor. High variance inflation factors for the dummy variables for discrete risk factors may point to multicollinearity, and partial confounding between risk factors was also automatically signaled. Following univariate analyses, only potential risk factors associated with the dependent variable $(P<0.10)$ were offered to bi- or multivariate models. Selection of risk factors was performed both by stepwise forward and stepwise backward selection. The criterion in backward and forward selection was the adjusted coefficient of determination $\left(\mathrm{R}^{2}\right)$. On the union of final models of both selection procedures, best subset selection was performed and significance tests for the effects of the selected risk factors were evaluated. Only risk factors that added significance $(P<0.05)$ to the model were retained. Risk factors that were (partially) confounded were not simultaneously maintained in the same model. No interactions between risk factors were considered.

For the analysis of prevalence (i.e., percentages per farm, or batch), logistic regression models were used, specifying a binomial variance function with a multiplicative dispersion parameter. Estimation was by quasi likelihood. The dispersion parameter in the variance was estimated from the data (McCullagh and Nelder, 1983). For each risk factor retained in a final model, odds ratios (OR) as well as $95 \%$ confidence intervals were obtained.

\section{RESULTS}

\section{Farm Environment and Management Data}

Reflecting the European veal calf production, the majority of farms included in the sample raised white veal in small groups with less than 16 calves/pen. Farms adopting large groups housed 26 to 50 calves/ pen. Calves were predominantly males; $70 \%$ of the batches included less than $5 \%$ of females. Holstein was the prevalent breed of calves, and raised in $66.5 \%$ of the 
farms. Pure dual-purpose breed calves were raised in $16.5 \%$ of the farms, whereas $17 \%$ of the farms fattened crosses between different dairy and beef breeds.

Considering the calf feeding plan, 3 levels were distinguished for the total amount of milk-replacer powder administered throughout the fattening cycle. Twenty percent of farms provided 280 to $330 \mathrm{~kg}$ of milk-replacer powder/calf during fattening; $51 \%$ of farms provided 331 to $380 \mathrm{~kg} /$ calf, and $29 \%$ of farms provided $>380$ $\mathrm{kg} /$ calf. Four levels were set for the total amount of solid feed delivered during the fattening cycle. Seventeen percent of farms provided a minimum amount of solid feed ( $\leq 50 \mathrm{~kg}$ of $\mathrm{DM} /$ calf); $23 \%$ provided 51 to $100 \mathrm{~kg}$ of DM/calf; $48 \%$ provided 101 to $150 \mathrm{~kg}$ of DM/ calf, whereas the remaining $12 \%$ of farms provided 151 to $300 \mathrm{~kg}$ of DM/calf. Drinking water was provided ad libitum in $27 \%$ of farms, it was restricted in $54 \%$ of farms, and it was not available for calves at any stage of the rearing cycle in $19 \%$ of farms. Calves were slaughtered within $30 \mathrm{wk}$ of fattening in the large majority of farms.

\section{Prevalence of Gastrointestinal Disorders Recorded During Postmortem Inspection}

On average, $59 \pm 10$ rumens and abomasa were inspected per farm. Regarding rumen development, a high prevalence of rumens with score 1 occurred, whereas the lowest percentage was scored 4 (Table 2). Rumen plaques were observed in $31 \%$ of inspected rumens and

Table 1. List of the tested potential risk factors for the occurrence of gastrointestinal disorders in veal calves

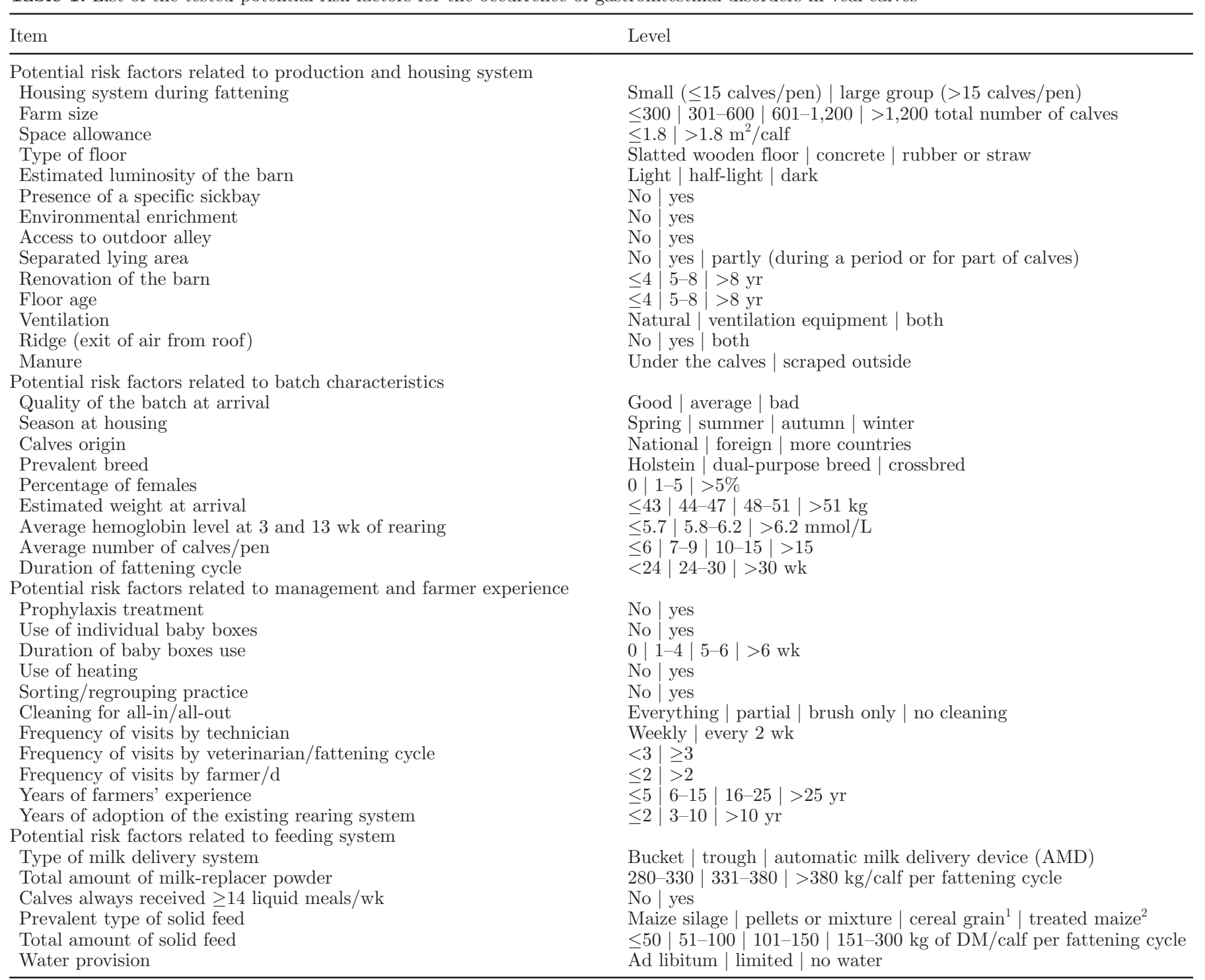

${ }^{1}$ Barley or maize.

${ }^{2}$ Rolled or flaked maize. 
Table 2. Descriptive statistics of data obtained during inspection carried out at the slaughterhouse on rumens and abomasa

\begin{tabular}{|c|c|c|c|c|}
\hline Item & Mean & SD & Minimum & Maximum \\
\hline \multicolumn{5}{|l|}{ Rumen development class, $\%$ of rumens } \\
\hline 1: Low & 60.4 & 32.25 & 0.0 & 100.0 \\
\hline 2: Moderate & 28.6 & 20.71 & 0.0 & 83.3 \\
\hline 3: Well & 9.9 & 17.63 & 0.0 & 76.9 \\
\hline 4: Full & 1.1 & 4.54 & 0.0 & 50.0 \\
\hline Rumen development mean score ${ }^{1}$ & 1.5 & 0.52 & 1.0 & 3.43 \\
\hline Rumen plaques, $\%$ of rumens & 31.3 & 26.16 & 0.0 & 100.0 \\
\hline Rumen hyperkeratinization, $\%$ of rumens & 6.1 & 8.15 & 0.0 & 46.7 \\
\hline \multicolumn{5}{|l|}{ Abomasal lesion, $\%$ of abomasa } \\
\hline Presence in pyloric region & 74.1 & 13.20 & 31.7 & 100.0 \\
\hline Presence on torus & 76.9 & 12.60 & 25.0 & 100.0 \\
\hline Abomasal lesions mean score ${ }^{2}$ & 4.3 & 3.24 & 1.0 & 19.8 \\
\hline
\end{tabular}

${ }^{1}$ Score calculated as mean of the scores of all of the rumens observed per batch.

${ }^{2}$ Score weighed according to the number of lesions (from 0 to maximum of 4 , censored) and size category $(1=$ $\left.<0.5 \mathrm{~cm}^{2} ; 2=0.5-1 \mathrm{~cm}^{2} ; 3=>1 \mathrm{~cm}^{2}\right)$ calculated as [(n of lesions size $\left.1 \times 1\right)+(\mathrm{n}$ of lesions size $2 \times 2)+(\mathrm{n}$ of lesions size $3 \times 3)$ ].

the prevalence of rumens with papillae hyperkeratosis was $6.1 \%$ (Table 2 ).

More than $70 \%$ of the observed abomasa showed at least one lesion in the pyloric area and a similar result was found for the torus pylorus, reaching in some batches the maximum of $100 \%$ of the inspected organs (Table 2).

\section{Risk Factor Analysis for Low Rumen Development}

Because low rumen development (score 1) was described in the literature as a relevant problem for conventional veal calves, risk factor analysis was restricted to this variable. The first one-way logistic regression analysis showed that potential risk factors were linked particularly to feeding and housing factors (Table 3 ). The OR for poor rumen development was almost 15 in farms delivering low amounts of solid feed $(\leq 50 \mathrm{~kg}$ of $\mathrm{DM} /$ calf per cycle) compared with those in which high quantities were supplied (151-300 kg of DM/calf per cycle). This variable alone explained almost $30 \%$ of the variance related to this problem. In farms where maize silage or pellets/mixture were used as the prevalent types of solid feed, compared with the use of cereal grain, the risk of low rumen development increased with an OR of 4.2 and 4.4, respectively. Considering the milk delivery system, the pairwise comparison between bucket and trough was not relevant, whereas the risk of low rumen development increased when these systems were compared with AMD. The absence of a dedicated sickbay in comparison with the presence of a sickbay, and the wooden slatted floor in comparison with the concrete floor also showed OR over 1 (Table 3).

Due to multicollinearity, the total amount and type of solid feed were not simultaneously included in the final multivariate regression model reported in Table
3. Looking at pairwise comparisons among the adopted classes for factors such as the total amount of milk powder and the water provision, OR for low rumen development were always below 1 , meaning that these classes diminished the risk for its occurrence. The risk of poor rumen development increased in farms with small group housing when compared with that in large groups. Regarding the season effect, farms in which animals were housed during the summer showed the lowest incidence of poor rumen development, whereas spring increased the risk compared with that in autumn and in summer in particular.

\section{Risk Factor Analysis for Occurrence of Rumen Plaques and Hyperkeratosis}

Significant risks for rumen plaques obtained from the one-way logistic regression analysis are shown in Table 4. Feeding low quantities of solid feed ( $\leq 50 \mathrm{~kg}$ of DM/ calf) decreased the risk of rumen plaques, whereas the provision of low amounts of milk powder (280-330 kg/ calf) increased it more than 10 times. Calves that did not receive at least 2 milk meals per day (feed restriction for $1 \mathrm{~d} / \mathrm{wk}$ ) showed a higher risk of plaques on the rumen wall compared with calves that were weekly fed 14 liquid meals or more. Farms that reared national calves increased the risk of developing rumen plaques compared with those that fattened animals imported from more countries. The OR for rumen plaques was above 1 in farms that did not use heating as well as in those not equipped with individual boxes to separate the penmates during the first 8 wk of the rearing cycle. Pens with a wooden slatted floor and the absence of a sickbay were instead protective factors.

The multivariate risk factor model showed an association between occurrence of rumen plaques and 
Table 3. One-way logistic regression analysis and multivariate regression model for low rumen development in veal calves

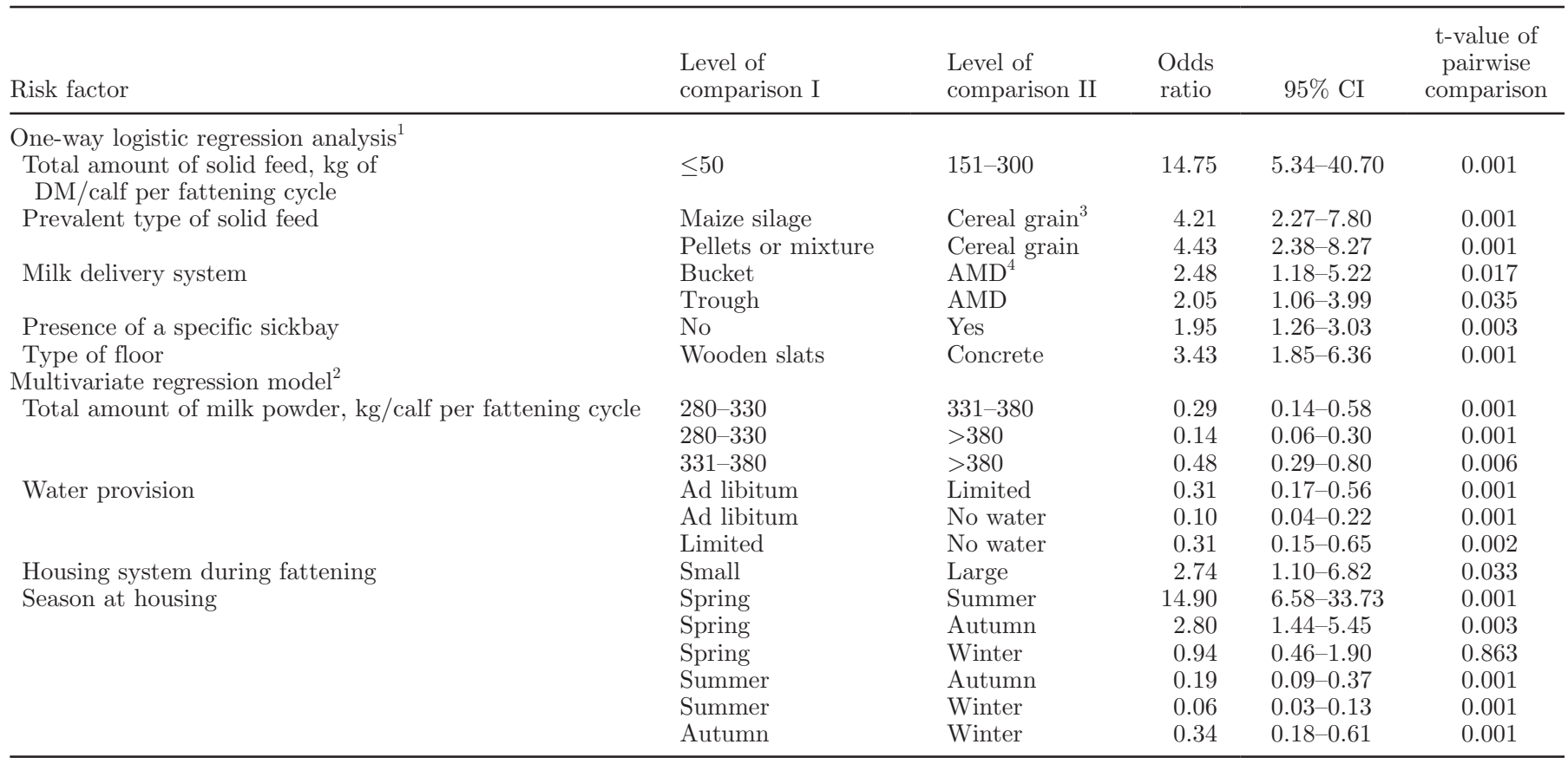

${ }^{1}$ Single risk factors in the one-way logistic regression analysis were significant at $P<0.05$.

${ }^{2}$ All risk factors in the multivariate regression model were significant at $P<0.05$ except for Housing system during fattening, which was not significant at $P=0.076$. Adjusted $\mathrm{R}^{2}=47.94 \%$.

${ }^{3}$ Barley or maize.

${ }^{4}$ Automatic milk delivery device.

type of solid feed, water provision, and space allowance (Table 4). According to OR, feeding cereal grain as the prevalent solid feed increased the risk of rumen plaques. Maize silage acted as a preventive factor when compared with pellets/mixture or cereal grain. Pairwise comparisons among the classes of water provision showed that ad libitum supply was a risk factor for rumen plaques. A space allowance $\leq 1.8 \mathrm{~m}^{2} /$ calf doubled the risk for rumen plaques compared with that of larger individual space availability.

One-way logistic regression analysis showed that bucket feeding compared with the use of a common trough decreased the risk of rumen papillae hyperkeratinization (Table 5). A further preventive measure was the administration of a total amount of solid feed $\leq 50 \mathrm{~kg}$ of $\mathrm{DM} /$ calf compared with greater amounts (151-300 kg of $\mathrm{DM} /$ calf). Holstein calves had a lower risk of developing hyperkeratinization of the papillae compared with that of crossbred animals, whereas a space allowance $\leq 1.8$ $\mathrm{m}^{2} /$ calf increased such risk (Table 5).

The final multivariate risk factor model for the occurrence of rumen hyperkeratosis considered prevalent type of solid feed and the presence of a dedicated sickbay (Table 5). Feeding cereal grain highly increased the risk of rumen papillae hyperkeratinization. Furthermore, the absence of a dedicated sickbay represented a risk.

\section{Risk Factor Analysis for Occurrence of Abomasal Lesions in the Pyloric Area}

Risk factors for occurrence of lesions in the pyloric area of veal calf abomasa were mainly linked to feeding strategies and water provision (Table 6). The provision of amounts of milk powder ranging from 280 to $330 \mathrm{~kg} /$ calf increased the risk of abomasal lesions compared with that from higher amounts, whereas an opposite result was observed for the amounts of solid feed. The ad libitum provision of drinking water increased the risk of abomasal lesions when compared with that from the absence of drinking water. No use of heating seemed to be an additional risk factor, whereas a lower frequency of visits by the veterinarian was a preventive measure.

The final multivariate regression model for abomasal lesions in the pyloric area showed that the prevalent type of solid feed and the season at housing were the most relevant factors explaining about $41 \%$ of the total variance related to this problem (Table 6). Pairwise comparisons among classes of solid feeds showed the highest risk of lesions in the pyloric area when calves were fed cereal grain. The risk of having at least one lesion on the pylorus was lower for calves housed during the spring, whereas it was higher for those housed during the summer and autumn compared with that during the winter. 
Table 4. One-way logistic regression analysis and multivariate regression model for the incidence of rumen plaques in veal calves

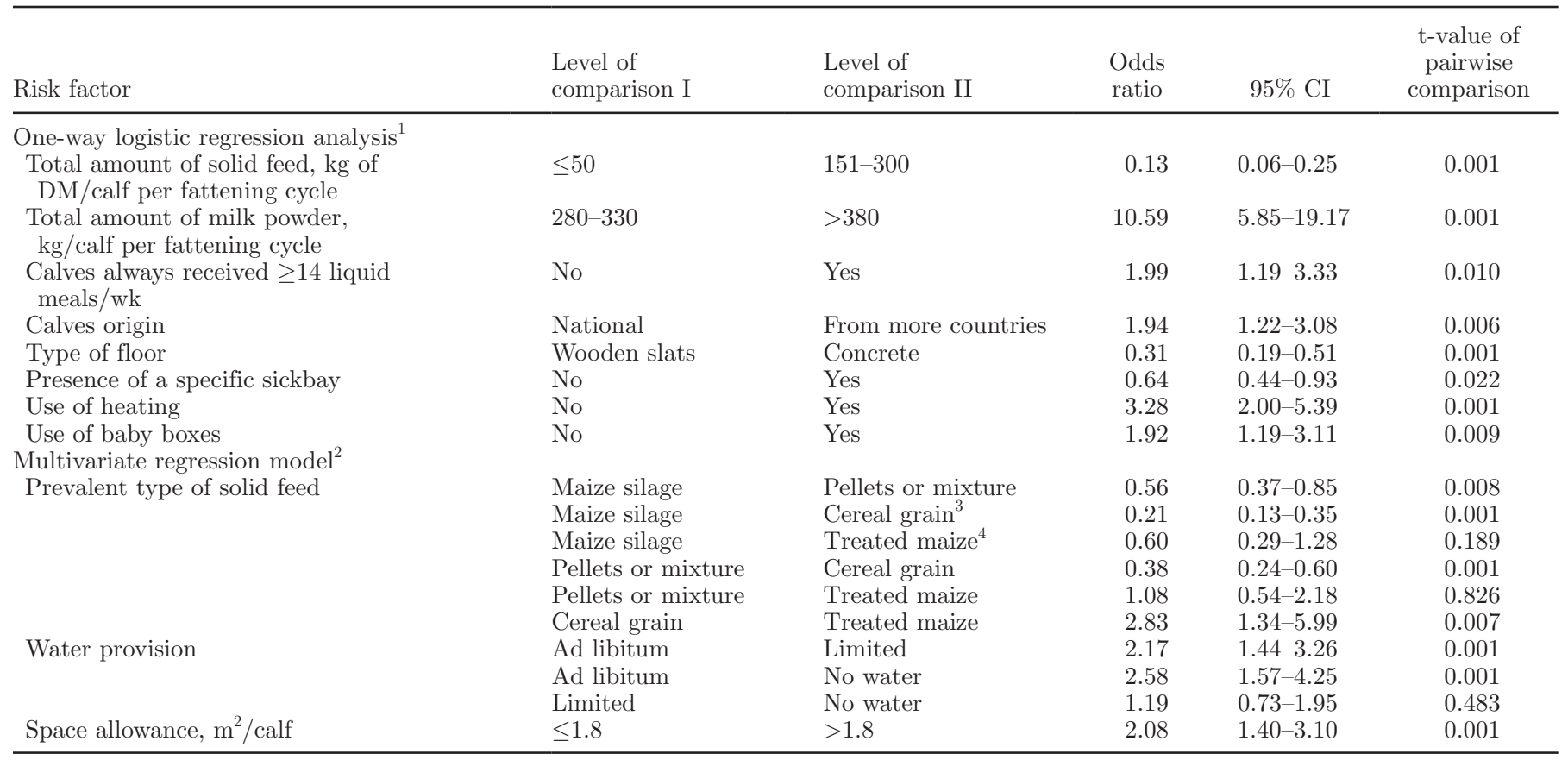

${ }^{1}$ Single risk factors in the one-way logistic regression analysis were significant for $P<0.05$.

${ }^{2}$ All risk factors in the multivariate regression model were significant at $P<0.001$. Adjusted $\mathrm{R}^{2}=31.51 \%$.

${ }^{3}$ Barley or maize.

${ }^{4}$ Rolled or flaked maize.

\section{DISCUSSION}

The gastrointestinal disorders investigated in this study might negatively affect the health and welfare status of veal calves by placing the animals in a borderline condition between pathological and physiological, where a physiological status indicates a developed and functioning rumen, an unaltered rumen mucosa, and a healthy abomasum. As expected, the occurrence of gastrointestinal disorders was mainly associated with feeding-related factors. Consistent with previous findings (Beharka et al., 1998; Cozzi et al., 2002a; Suárez et al., 2006b), the importance of supplementing veal calves with solid feed to stimulate rumen develop-

Table 5. One-way logistic regression analysis and multivariate regression model for the incidence of rumen papillae hyperkeratinization in veal calves

\begin{tabular}{|c|c|c|c|c|c|}
\hline Risk factor & $\begin{array}{l}\text { Level of } \\
\text { comparison I }\end{array}$ & $\begin{array}{l}\text { Level of } \\
\text { comparison II }\end{array}$ & $\begin{array}{l}\text { Odds } \\
\text { ratio }\end{array}$ & $95 \% \mathrm{CI}$ & $\begin{array}{c}\text { t-value of } \\
\text { pairwise } \\
\text { comparison }\end{array}$ \\
\hline \multicolumn{6}{|l|}{ One-way logistic regression analysis ${ }^{1}$} \\
\hline Milk delivery system & Bucket & Trough & 0.32 & $0.18-0.57$ & 0.001 \\
\hline $\begin{array}{l}\text { Total amount of solid feed, } \mathrm{kg} \text { of } \\
\text { DM/calf per fattening cycle }\end{array}$ & $\leq 50$ & $151-300$ & 0.38 & $0.16-0.90$ & 0.030 \\
\hline Prevalent breed & Holstein & Crossbred & 0.50 & $0.32-0.79$ & 0.003 \\
\hline Space allowance, $\mathrm{m}^{2} /$ calf & $\leq 1.8$ & $>1.8$ & 2.72 & $1.64-4.52$ & 0.001 \\
\hline \multicolumn{6}{|l|}{ Multivariate regression model $^{2}$} \\
\hline \multirow[t]{6}{*}{ Prevalent type of solid feed } & Maize silage & Pellets or mixture & 0.82 & $0.53-1.26$ & 0.372 \\
\hline & Maize silage & Cereal grain ${ }^{3}$ & 0.40 & $0.25-0.63$ & 0.001 \\
\hline & Maize silage & Treated maize ${ }^{4}$ & 35.34 & $0.60-2,068.66$ & 0.088 \\
\hline & Pellets or mixture & Cereal grain & 0.49 & $0.31-0.76$ & 0.002 \\
\hline & Pellets or mixture & Treated maize & 43.03 & $0.73-2,520.38$ & 0.072 \\
\hline & Cereal grain & Treated maize & 88.53 & $1.51-5,196.27$ & 0.032 \\
\hline Presence of a specific sickbay & No & Yes & 1.66 & $1.12-2.44$ & 0.012 \\
\hline
\end{tabular}

${ }^{1}$ Single risk factors in the one-way logistic regression analysis were significant at $P<0.05$.

${ }^{2}$ All risk factors in the multivariate regression model were significant at $P<0.05$. Adjusted $\mathrm{R}^{2}=17.64 \%$.

${ }^{3}$ Barley or maize.

${ }^{4}$ Rolled or flaked maize. 
Table 6. One-way logistic regression analysis and multivariate regression model for the prevalence of abomasal lesions (at least one lesion, from superficial scar to ulcer) in the pyloric area in veal calves

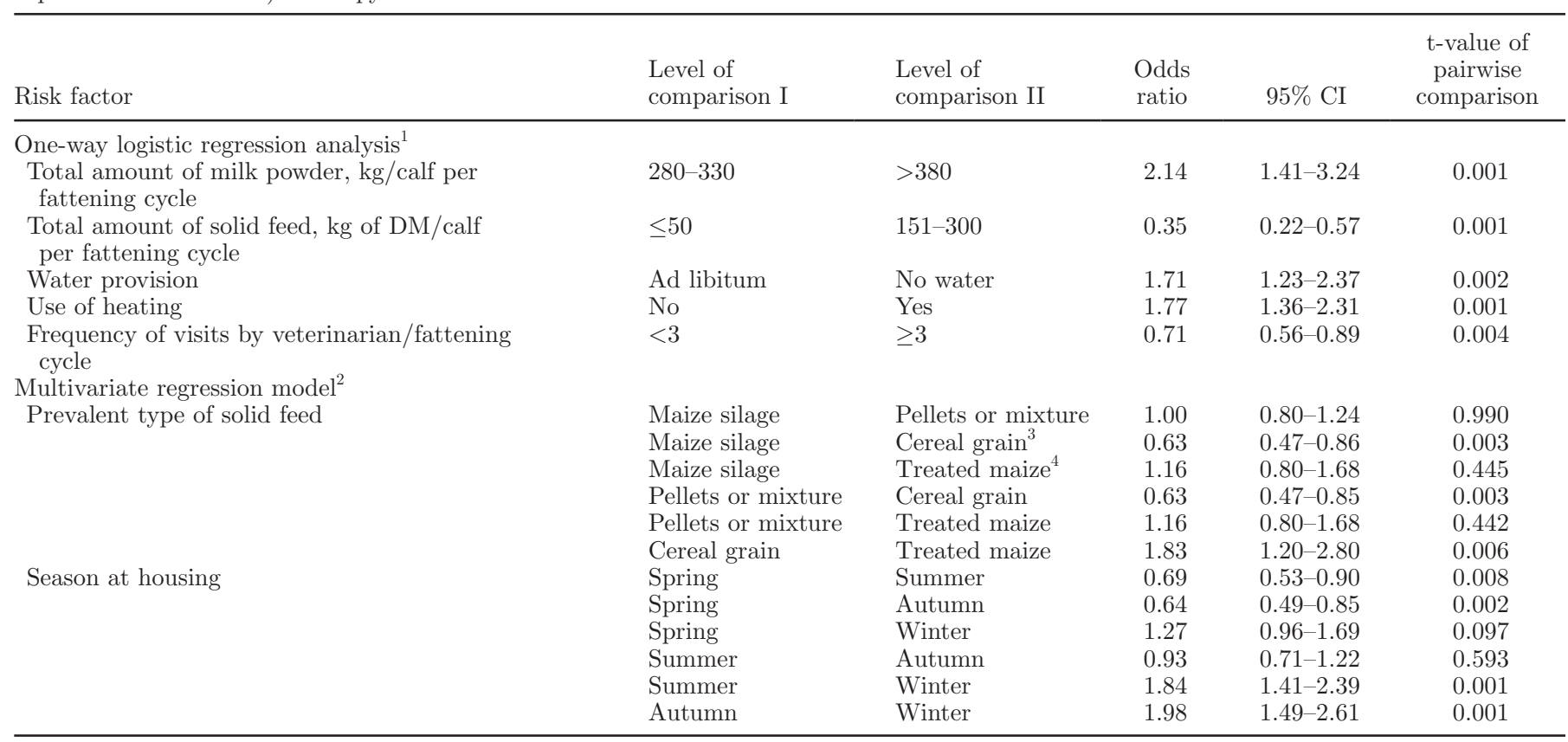

${ }^{1}$ Single risk factors in the one-way logistic regression analysis were significant at $P<0.05$.

${ }^{2}$ All risk factors in the multivariate regression model were significant at $P<0.004$. Adjusted $\mathrm{R}^{2}=40.79 \%$.

${ }^{3}$ Barley or maize.

${ }^{4}$ Rolled or flaked maize.

ment has been demonstrated. However, results of the present study would suggest increasing the amount of solid feed provided during the fattening cycle far above the recommended 50 to $250 \mathrm{~g} /$ calf per day set by the European Union regulation. Forestomach size innately increases with age, but its papillae development requires physical stimulation and VFA (mainly butyrate and propionate) from microbial fermentation of solid feedstuffs (Beharka et al., 1998). Compared with maize silage or pellets that have a relatively high content of fiber, a starchy substrate, such as cereal grain, has been shown to stimulate rumen development, likely through a higher production of butyrate and propionate (Sander et al., 1959). Results of the multivariate regression model revealed the preventive effect on low rumen development of reducing the total amount of milk replacer fed throughout fattening. This finding might still be related to the provision of solids which commonly increases in feeding plans based on a restricted amount of milk powder (Suárez et al., 2007). A gradual reduction of the milk replacer associated with availability of increasing amounts of solid feed would reflect a more natural condition for calves, promoting the changes from a preruminant into a ruminant animal. The increased risk for poor rumen development associated with the use of buckets or troughs for milk delivery compared with that from use of AMD could again be linked to the solid feed distribution. Where milk is automatically delivered, usually solids are also distributed by computer-controlled feeders. These devices allow a more balanced intake of solid feed among all penmates, avoiding that a few dominant calves eat a large amount of solid feed as in small-group systems where feeds are delivered in common mangers. The outcomes of the multivariate regression model seem to support this hypothesis because the risk for low rumen development has been shown to be increased in calves housed in small groups.

It is interesting to notice that if the results for poor rumen development would recommend increasing the amount of solid feed for veal calves, those regarding the occurrence of rumen plaques and papillae hyperkeratinization suggest an opposite strategy. Moreover, whereas the prevalent provision of cereal grain promoted rumen development, it was a significant risk factor for rumen plaques and hyperkeratosis as well as for abomasal lesions. These controversial findings, with the same variable acting as preventive or predisposing factor for different disorders, demonstrate the difficulty of identifying from the present data the best feeding options capable of minimizing the prevalence of all the considered gastrointestinal disorders.

Results for plaques are consistent with the findings of Suárez et al. (2006b) who found out that supplementing 
veal calves with concentrates increased the weight of the empty rumen and mucosa thickness but it enhanced the incidence of rumen plaques. The association of cereal grain and hyperkeratosis could arise either from the lack of abrasive effect of this type of feed compared with that of roughage sources (Greenwood et al., 1997; Beharka et al., 1998) or from the altered rumen fermentation profile with high propionate concentration (Hinders and Owen, 1965; Bertram et al., 2009). In both cases, a calf might be more easily subject to a potential (sub)acidosis due to the lower rumen-buffering capacity and the subsequent decrease in rumen $\mathrm{pH}$ (Anderson et al., 1982), with detrimental effects on its health and welfare status. The straight link between an increment of solid feed intake and the consumption of drinking water when water was provided ad libitum to preweaned calves (Kertz et al., 1984), could explain the results of our study, in which ad libitum drinking water availability acted as a risk factor for rumen plaques. No clear explanation for the association between space allowance and rumen plaques and hyperkeratosis exists except that providing calves with a larger space allowance than that set by the legal requirement $\left(1.8 \mathrm{~m}^{2} /\right.$ calf with $\mathrm{BW} \geq 220 \mathrm{~kg}$ ) may constitute a further strategy to limit their stress and to improve their welfare, possibly with beneficial effects on the health status of the rumen. On the one hand, our results suggest that the etiology of rumen mucosa alterations is not restricted to factors related to the feeding plan but may also involve other risk factors to which veal calves are exposed during rearing. On the other hand, they should encourage the development and application on-farm of further housing and management solutions with the aim at improving calf well-being.

In the present study, more than $74 \%$ of the inspected abomasa showed at least one lesion and this high prevalence is consistent with data of previous studies (Gottardo et al., 2002; Mattiello et al., 2002). This result appears lower than that from Welchman and Baust (1987) who reported that $97 \%$ of calves housed in individual cages and fed a traditional all-liquid diet suffered from abomasal ulcers. This might suggest that some improvements have been achieved by the actual systems of veal calf production but also that the predisposing factors for abomasal damage are still not overcome. The recent advance in veal calf management did not modify the way milk replacer is provided to veal calves throughout the entire fattening cycle, with large volumes of liquids fed usually in a few meals per day. Regardless of the type of milk replacer, this could be the main critical point for abomasal distension and its subsequent ulceration, particularly when compared with the physiological nursing behavior of the suckling calf consisting of 5 to 9 bouts/d (Day et al., 1987). Unexpectedly, in the current study, amounts of milk powder ranging from 280 to $330 \mathrm{~kg} /$ calf per fattening cycle increased the risk of occurrence of abomasal lesions compared with that from greater amounts. A possible reason for this result might be linked to greater amounts of solid feed provided in farms adopting low levels of milk replacer. Under this latter feeding plan, solids may promote abomasal damage by worsening the mechanical load of the stomach. According to the literature, lesions seem to be generally associated with coarse solid feed (Cozzi et al., 2002b; Mattiello et al., 2002; Bähler et al., 2010), but the outcomes of the present risk analysis showed that cereal grain was also a risk factor. Differing from the results of Bähler et al. (2010), no associations were found between abomasal lesions and the type of milk delivery system. Free access to water was a further risk factor in veal farms, but this is in contradiction with results of Gottardo et al. (2002) who found no effect and Bähler et al. (2010) who reported an OR of 2.3 for lesions in the fundic area when no water was provided. Similar to rumen problems, results suggest that, in addition to risk factors related to feeding, housing and management factors may also play a role in the etiology of abomasal lesions.

\section{CONCLUSIONS}

This cross-sectional study was carried out on a large sample of farms in the 3 main veal meat-producing countries in Europe to gather reliable information on the prevalence of gastrointestinal disorders and the main risk factors related to their occurrence. The coming-inforce of the European regulation for calf protection has promoted relevant improvements of calf behavior and health, but the results of the current study have shown that gastrointestinal disorders are still highly prevalent. Factors related to the feeding system were confirmed to be the main risks for the occurrence of poor rumen development and of the alterations of rumen and abomasal mucosa. However, their etiology should consider other predisposing factors related to housing and management because some options adopted to improve calf welfare were associated with lower risks. Finally, controversial findings, where the same variable acted as a preventive or predisposing factor for different disorders, demonstrate the difficulty in identifying the best rearing options capable of inducing a sharp decrease in the prevalence of all of the considered gastrointestinal disorders.

\section{ACKNOWLEDGMENTS}

The study was carried within the activity of the European Union-funded integrated project FOODCT-2004-506508 "Welfare Quality: Science and society improving animal welfare in the food quality chain." 
The text represents the authors' viewpoint and does not necessarily represent the position of the Consortium, which will not be liable for the use made of such information. This study was also partially supported by Italian veal calf producers, the French veal product board (Interveaux), the Dutch Ministry of Agriculture, Nature and Food Quality, Dutch Product Board for Animal Feed, and Dutch Product Boards for Livestock, Meat, and Eggs. The authors thank the veal producers who took part in the study, Willem Buist (Wageningen University \& Research Centre, Biometrics, Wageningen, the Netherlands) for his help with the data analysis, and Hélène Leruste (Groupe ISA, Lille Cedex, France), Roberto Chimetto (Department of Animal Science, University of Padova, Legnaro, Italy), Roberto Rampazzo (Department of Animal Science, University of Padova), Salvatore Franco (Azienda ULSS 15 Alta Padovana, Cittadella, Italy), Paola Prevedello (Department of Animal Science, University of Padova), and Elena Tessitore (Department of Animal Science, University of Padova) for their cooperation in the data collection.

\section{REFERENCES}

Anderson, M. J., M. Khoyloo, and J. L. Walters. 1982. Effect of feeding whole cottonseed on intake, body weight, and reticulorumen development of young Holstein calves. J. Dairy Sci. 65:764-772.

Andrighetto, I., F. Gottardo, D. Andreoli, and G. Cozzi. 1999. Effect of type of housing on veal calf growth performance, behaviour and meat quality. Livest. Prod. Sci. 57:137-145.

Babu, L. K., H. N. Pandey, and A. Sahoo. 2004. Effect of individual versus group rearing on ethological and physiological responses of crossbred calves. Appl. Anim. Behav. Sci. 87:177-191.

Bähler, C., G. Regula, M. H. Stoffel, A. Steiner, and A. von Rotz. 2010. Effects of the two production programs 'Naturafarm' and 'conventional' on the prevalence of non-perforating abomasal lesions in Swiss veal calves at slaughter. Res. Vet. Sci. 88:352-360.

Beharka, A. A., T. G. Nagaraja, J. L. Morrill, G. A. Kennedy, and R. D. Klemm. 1998. Effects of form of the diet on anatomical, microbial, and fermentative development of the rumen of neonatal calves. J. Dairy Sci. 81:1946-1955.

Bertram, H. C., N. B. Kristensen, M. Vestergaard, S. K. Jensen, J. Sehested, N. C. Nielsen, and A. Malmendal. 2009. Metabolic characterization of rumen epithelial tissue from dairy calves fed different starter diets using H-1 NMR spectroscopy. Livest. Sci. 120:127-134.

Bokkers, E. A. M., and P. Koene. 2001. Activity, oral behaviour and slaughter data as welfare indicators in veal calves: A comparison of three housing systems. Appl. Anim. Behav. Sci. 75:1-15.

Broom, D. M. 1991. Needs and welfare of housed calves. Pages 23-31 in New Trends in Veal Calf Production. EAAP Publication no. 52. J. H. M. Metz and C. M. Groenestein, ed. EAAP, Wageningen, the Netherlands.

Cozzi, G., F. Gottardo, S. Mattiello, E. Canali, E. Scanziani, M. Verga, and I. Andrighetto. 2002a. The provision of solid feeds to veal calves: I. Growth performance, forestomach development, carcass and meat quality. J. Anim. Sci. 80:357-366.

Cozzi, G., F. Gottardo, F. Mutinelli, B. Contiero, G. Fregolent, S. Segato, and I. Andrighetto. 2002b. Growth performance, behaviour, forestomach development and meat quality of veal calves provided with barley grain or ground wheat straw for welfare purpose. Ital. J. Anim. Sci. 1:113-126.

Day, M. L., K. Imakawa, A. C. Clutter, P. L. Wolfe, D. D. Zalesky, M. K. Nielsen, and J. E. Kinder. 1987. Suckling behavior of calves with dams varying in milk production. J. Anim. Sci. 65:1207-1212.
Di Giancamillo, A., G. Bosi, S. Arrighi, G. Savoini, and C. Domeneghini. 2003. The influence of different fibrous supplements in the diet on ruminal histology and histometry in veal calves. Histol. Histopathol. 18:727-733.

European Council. 1991. Directive 91/629/EC laying down minimum standards for the protection of calves. Official Journal of the European Communities of 11 December 1991, No. L 340/28.

European Council. 1997. Directive 97/2/EC amending Directive 91/629/EEC laying down minimum standards for the protection of calves. Official Journal of the European Communities of 28 January 1997 , No. L $25 / 24$.

EUROSTAT. 2007. Accessed Apr. 12, 2010. http://epp.eurostat. ec.europa.eu/cache/ITY_SDDS/en/apro_mt_p_esms.htm

GenStat Committee. 2000. Reference Manual. Procedure Library PL12, VSN International, Oxford, UK.

Gillman, C. E., A. L. KilBride, P. Ossent, and L. E. Green. 2009. A cross-sectional study of the prevalence of foot lesions in postweaning pigs and risks associated with floor type on commercial farms in England. Prev. Vet. Med. 91:146-152.

Gottardo, F., S. Mattiello, G. Cozzi, E. Canali, E. Scanziani, L. Ravarotto, V. Ferrante, M. Verga, and I. Andrighetto. 2002. The provision of drinking water to veal calves for welfare purpose. J. Anim. Sci. 80:2362-2372.

Greenwood, R. H., J. L. Morrill, E. C. Titgemeyer, and G. A. Kennedy. 1997. A new method of measuring diet abrasion and its effect on the development of the forestomach. J. Dairy Sci. 80:2534-2541.

Hinders, R. G., and F. G. Owen. 1965. Relation of ruminal parakeratosis development to volatile fatty acid absorption. J. Dairy Sci. 48:1069-1073.

Kertz, A. F., L. F. Reutzel, and J. H. Mahoney. 1984. Ad libitum water intake by neonatal calves and its relationship to calf starter intake, weight gain, feces score, and season. J. Dairy Sci. 67:2964-2969.

Mattiello, S., E. Canali, V. Ferrante, M. Caniatti, F. Gottardo, G Cozzi, I. Andrighetto, and M. Verga. 2002. The provision of solid feeds to veal calves: II. Behavior, physiology and abomasal damage. J. Anim. Sci. 80:367-375.

McCullagh, P., and J. A. Nelder. 1983. Generalized Linear Models. Chapman and Hall, London, UK.

Morisse, J. P., D. Huonnic, J. P. Cotte, and A. Martrenchar. 2000. The effect of four fibrous feed supplementations on different welfare traits in veal calves. Anim. Feed Sci. Technol. 84:129-136.

Welfare Quality Consortium. 2009. Welfare Quality Assessment Protocol for Cattle. Welfare Quality Consortium, Lelystad, the Netherlands.

Sander, E. G., R. G. Warner, H. N. Harrison, and J. K. Loosli. 1959. The stimulatory effect of sodium butyrate and sodium propionate on the development of rumen mucosa in the young calf. J. Dairy Sci. 42:1600-1605.

Suárez, B. J., C. G. Van Reenen, G. Beldman, J. van Delen, J. Dijkstra, and W. J. Gerrits. 2006a. Effects of supplementing concentrates differing in carbohydrate composition in veal calf diets: I. Animal performance and rumen fermentation characteristics. J. Dairy Sci. 89:4365-4375.

Suárez, B. J., C. G. Van Reenen, W. J. Gerrits, N. Stockhofe, A. M. van Vuuren, and J. Dijkstra. 2006b. Effects of supplementing concentrates differing in carbohydrate composition in veal calf diets: II. Rumen development. J. Dairy Sci. 89:4376-4386.

Suárez, B. J., C. G. van Reenen, N. Stockhofe, J. Dijkstra, and W. J. J. Gerrits. 2007. Effect of roughage source and roughage to concentrate ratio on animal performance and rumen development in veal calves. J. Dairy Sci. 90:2390-2403.

Veissier, I., A. Boissy, A. M. dePassillé, J. Rushen, C. G. van Reenen, S. Roussel, S. Andanson, and P. Pradel. 2001. Calves' responses to repeated social regrouping and relocation. J. Anim. Sci. 79:2580-2593.

Welchman, D. D., and G. N. Baust. 1987. A survey of abomasal ulceration in veal calves. Vet. Rec. 121:586-590.

Wiepkema, P. R., K. K. van Hellemond, P. Roessingh, and H. Romberg. 1987. Behaviour and abomasal damage in individual veal calves. Appl. Anim. Behav. Sci. 18:257-268. 